\title{
A TAUBERIAN THEOREM FOR STRONG ABEL SUMMABILITY TYPE
}

\author{
INDULATA SUKLA
}

\begin{abstract}
In the present paper the author has defined a new method of strong Abel summability type $\{A, \lambda\}_{m}$ and obtained a necessary and sufficient type of Tauberian conditions for $\Sigma a_{n}$ to be summable $[R, \lambda, k]_{m}$, whenever it is summable $\{A, \lambda\}_{m}$. This result is analogous to one result of Flett [4].
\end{abstract}

1. Let $\left\{\lambda_{n}\right\}$ be an increasing sequence of nonnegative numbers tending to $\infty$ with $n$. The series $\Sigma a_{n}$ is said to be strongly summable $[R, \lambda, k]_{m}, k>1-\frac{1}{m}$, to the sum $s[5,8]$, if

$$
\int_{\lambda_{0}}^{x}\left|c_{\lambda}^{k-1}(t)-s\right|^{m} d t=o(x), \quad \text { as } x \rightarrow \infty,
$$

where $c_{\lambda}^{k}(x)=\Sigma_{\lambda_{0}<x}\left(1-\lambda_{v} / x\right)^{k} a_{v}$.

It is noteworthy to remark that in [5], Glatfeld uses a different notation.

We also write

$$
A_{\lambda}^{k}(x)=\sum_{\lambda_{n}<x}\left(x-\lambda_{n}\right)^{k} a_{n}, \quad B_{\lambda}^{k}(x)=\sum_{\lambda_{n}<x}\left(x-\lambda_{n}\right)^{k} \lambda_{n} a_{n} .
$$

It is natural to define the series $\sum a_{n}$ as summable $\{A, \lambda\}_{m}(m \geqslant 1)$ if the series $\phi(x)=\sum_{n=0}^{\infty} a_{n} \exp \left(-\lambda_{n} x\right)$ converges for all $x>0$ and

$$
\int_{R}^{\infty} \frac{|\phi(x)-s|^{m}}{\left(1-e^{-x}\right)^{2}} e^{-x} d x=o\left(\frac{1}{1-e^{-R}}\right), \quad \text { as } R \rightarrow 0 .
$$

For in the special case $\lambda_{n}=n$ this reduces after an obvious change of variable, to the definition of $\{A\}_{m}$ given by Flett in [4]. However we can put this definition in a simpler form. In fact, (1.2) is equivalent to the assertions that as $R \rightarrow 0+$,

$$
\int_{R}^{\infty} \frac{|\phi(x)-s|^{m}}{x^{2}} d x=o\left(\frac{1}{R}\right) .
$$

To prove this, we first note that, as $R \rightarrow 0+, 1 /\left(1-e^{-R}\right) \sim \frac{1}{R}$ so that $o\left(1 /\left(1-e^{-R}\right)\right)$ on the right of (1.2) can be replaced by $o\left(\frac{1}{R}\right)$. Next, the assumption that $\sum_{n=0}^{\infty} a_{n} \exp \left(-\lambda_{n} x\right)$ converges for $x>0$ implies that the sum is bounded for $x>1$ (see [6]). Hence the integrals

$$
\int_{1}^{\infty} \frac{|\phi(x)-s|^{m}}{\left(1-e^{-x}\right)^{2}} e^{-x} d x, \quad \int_{1}^{\infty} \frac{|\phi(x)-s|^{m}}{x^{2}} d x
$$

Received by the editors March 1, 1980.

1980 Mathematics Subject Classification. Primary 40E05; Secondary 40F05, 40G05.

() 1982 American Mathematical Society 0002-9939/82/0000-0407/\$02.75 
converge. Thus, since these integrals do exist, they are constants and hence certainly $o\left(\frac{1}{R}\right)$, so that it makes no difference to the validity of either (1.2) or (1.3) if we replace the integrals from $R$ to $\infty$ by integrals from $R$ to 1 . But, for $0<x<1$,

$$
\alpha / x^{2} \leqslant e^{-x} /\left(1-e^{-x}\right)^{2}<\beta / x^{2},
$$

where $\alpha, \beta$ are strictly positive absolute constants. Hence, for $0<R<1$,

$$
\alpha \int_{R}^{1} \frac{|\phi(x)-s|^{m}}{x^{2}} d x \leqslant \int_{R}^{1} \frac{|\phi(x)-s|^{m}}{\left(1-e^{-x}\right)^{2}} e^{-x} d x \leqslant \beta \int_{R}^{1} \frac{|\phi(x)-s|^{m}}{x^{2}} d x,
$$

so that if either one of the integrals occurring in (1.4) is $o\left(\frac{1}{R}\right)$ then so is the other proving the result.

Remark. The equivalent definition (1.3) and the above proof is due to $B$. Kuttner. $[c, k]_{m}$.

Hyslop and Boyd [1] have shown that for $\lambda_{n}=n,[R, \lambda, k]_{m}$ is equivalent to

Throughout we denote by $M$ a positive constant, which may be different at each occurrence.

2. Introduction. Flett [3] has established that $\sum_{n-1}^{r}\left|\tau_{n}^{k}\right|^{m}=o(r)$ is a Tauberian condition for $\sum a_{n} \in\{A\}_{m}$ to imply $\sum a_{n} \in[c, k]_{m}, m>1$ and $k>0$. The object of the present paper is to obtain an analogue of Flett's Theorem [3] and get a necessary and sufficient type of Tauberian condition for $\Sigma a_{n} \in\{A, \lambda\}_{m}$ to imply $\sum a_{n} \in[R, \lambda, k]_{m}$.

3. We establish the following.

THEOREM. If $m \geqslant 1$ and $k>1-\frac{1}{m}$ and (i) $\sum_{n=0}^{\infty} a_{n}$ is summable $\{A, \lambda\}_{m}$, then $a$ necessary and sufficient condition for $\sum_{n=0}^{\infty} a_{n}$ to be summable $[R, \lambda, k]_{m}$ is

$$
\int_{0}^{X}\left|\frac{B_{\lambda}^{k-1}(x)}{x^{k}}\right|^{m} d x=o(X), \quad \text { as } X \rightarrow \infty \text {. }
$$

4. We require the following lemmas.

LEMMA 1 [2]. If $\phi(x)=\sum_{n=0}^{\infty} a_{n} \exp \left(-\lambda_{n} x\right)$ converges for $x>0$, then for $k>0$, we have

$$
\phi(x)=\frac{x^{k+1}}{\Gamma(k+1)} \int_{0}^{\infty} A_{\lambda}^{k}(t) e^{-x t} d t .
$$

LEMMA 2. For $\sum a_{n}$ to be summable $[R, \lambda, k]_{m}$ it is necessary and sufficient that (i) $\sum a_{n}$ is summable $[R, \lambda, k+1]_{m}$ and (ii) (3.1) holds.

Proof. Let $m \geqslant 1$. Suppose first that (i) and (ii) are satisfied. We have [7]

$$
x^{-k+1} A_{\lambda}^{k-1}(x)=x^{-k} A_{\lambda}^{k}(x)+x^{-k} B_{\lambda}^{k-1}(x) \text {. }
$$

Hence

$$
\int_{0}^{X}\left|c_{\lambda}^{k-1}(x)\right|^{m} d x \leqslant M \int_{0}^{X}\left|c_{\lambda}^{k}(x)\right|^{m} d x+M \int_{0}^{X}\left|\frac{B_{\lambda}^{k-1}(x)}{x^{k}}\right|^{m} d x
$$


Since $[R, \lambda, k]_{m} \Rightarrow[R, \lambda, k+1]_{m}$ [7], (i) is necessary. Again by (4.1), (3.1) follows. Hence (3.1) is necessary.

LEMMA 3. If $m \geqslant 1, f(x)=f(x, t)$ and $g(x)=g(x, t)$ then

$$
\begin{aligned}
\left(\int d t \int|f(x)+s(x)|^{m} d x\right)^{1 / m}< & \left(\int d t \int|f(x)|^{m} d x\right)^{1 / m} \\
& +\left(\int d t \int|g(x)|^{m} d x\right)^{1 / m} .
\end{aligned}
$$

The result follows by double applications of Minkowski's inequality.

LEMMA 4. If $k>-1$, then

$$
\int_{u}^{\infty} v^{k} e^{-t v} d v=O\left(\frac{u^{k} e^{-t u}}{t}\right)+O\left(\frac{e^{-t u}}{t^{k+1}}\right),
$$

uniformly for all $t>0$ and $u \geqslant 0$.

Proof. If $k$ is any real constant, then uniformly in $y \geqslant 1$,

$$
\int_{y}^{\infty} w^{k} e^{-w} d w=O\left(y^{k} e^{-y}\right)
$$

This may be proved by observing that for sufficiently large $y$, say $y>Y$,

$$
y^{k} e^{-y}=-\int_{y}^{\infty} \frac{d}{d w}\left(w^{k} e^{-w}\right) d w \geqslant c \int_{y}^{\infty} w^{k} e^{-w} d w
$$

where $c$ is a strictly positive constant. But for fixed $Y,(4.2)$ certainly holds in the range $1 \leqslant y \leqslant Y$, since, in that range, $\int_{y}^{\infty} w^{k} e^{-w} d w$ and $y^{k} e^{-y}$ each lies between two (strictly) positive constants. By an obvious change of variables, we deduce that, uniformly in $t u \geqslant 1$,

$$
\int_{u}^{\infty} v^{k} e^{-t v} d v=O\left(\frac{u^{k} e^{-t u}}{t}\right)
$$

Let us now suppose $k>-1$. Then

$$
\int_{u}^{\infty} v^{k} e^{-t v} d v \leqslant \int_{0}^{\infty} v^{k} e^{-t v} d v=\frac{\Gamma(k+1)}{t^{k+1}}
$$

If $t u<1$ then $1 \leqslant e \cdot e^{-t u}$ and hence, uniformly in $t u<1$,

$$
\int_{u}^{\infty} v^{k} e^{-t v} d v=O\left(\frac{e^{-t u}}{t^{k+1}}\right) .
$$

Since (4.3) holds for $t u \geqslant 1$ and (4.4) for $t u \leqslant 1$ we have uniformly for all $t>0$, $u>0$,

$$
\int_{u}^{\infty} v^{k} e^{-t v} d v=O\left(\frac{u^{k} e^{-t u}}{t}\right)+O\left(\frac{e^{-t u}}{t^{k+1}}\right)
$$

LEMMA 5. If $0<m \mu<1$, then (3.1) implies

$$
\int_{0}^{X} x^{-m \mu}\left|\frac{B_{\lambda}^{k-1}(x)}{x^{k}}\right|^{m} d x=o\left(X^{1-m \mu}\right) .
$$


Proof. It follows by integration by parts.

5. Proof of the theorem. Let us suppose without loss of generality $s=0$. The necessity part follows from Lemma 2 . For sufficiency, it is required to prove, by Lemma 3 that (1.3) with $s=0$ and (3.1) together imply

$$
\int_{0}^{X}\left|c_{\lambda}^{k}(x)\right|^{m} d x=o(X), \quad \text { as } X \rightarrow \infty \text {. }
$$

We take throughout $R=\frac{1}{x}$ so that as $R \rightarrow 0+$, we have

$$
\frac{1}{e} \int_{0}^{x}\left|c_{\lambda}^{k}(x)\right|^{m} d x \leqslant \int_{R}^{\infty} d t \int_{0}^{x}\left|c_{\lambda}^{k}(x)\right|^{m} x e^{-t x} d x .
$$

Hence by Lemma 3, if $m>1$ and trivially if $m=1$, the conclusion will follow if we prove that

$$
\begin{gathered}
\int_{R}^{\infty}|\phi(t)|^{m} d t \int_{0}^{X} x e^{-t x} d x=o(X) \\
\int_{R}^{\infty}\left|\phi(t)-c_{\lambda}^{k}(x)\right|^{m} \int_{0}^{X} x e^{-t x} d x=o(X) .
\end{gathered}
$$

Since the inner integral in (5.2) is less than $\int_{0}^{\infty} x e^{-t x} d x=1 / t^{2},(5.2)$ follows at once from (1.3). So we have to consider only (5.3). Now by Lemma 1 and Hölder's inequality,

$$
\begin{aligned}
\left|c_{\lambda}^{k}(x)-\phi(t)\right|^{m} \leqslant & M t^{k+1}\left(\int_{0}^{x} v^{k}\left|c_{\lambda}^{k}(x)-c_{\lambda}^{k}(v)\right|^{m} e^{-t v} d v\right) \\
& +M t^{k+1}\left(\int_{x}^{\infty} v^{k}\left|c_{\lambda}^{k}(x)-c_{\lambda}^{k}(v)\right|^{m} e^{-t v} d v\right) \\
& =S(x, t)+T(x, t) .
\end{aligned}
$$

If $v<x$, we have (by Hölder's inequality when $m>1$ and trivially when $m=1$ )

$$
\left|c_{\lambda}^{k}(x)-c_{\lambda}^{k}(v)\right|^{m}=M\left|\int_{v}^{x} \frac{B_{\lambda}^{k-1}(u)}{u^{k+1}} d u\right|^{m} \leq \frac{M}{v} \int_{v}^{x}\left|\frac{B_{\lambda}^{k-1}(u)}{u^{k}}\right|^{m} d u .
$$

Since $k>0$, using (5.5) we have

$$
S(x, t) \leqslant M t \int_{0}^{X}\left|\frac{B_{\lambda}^{k-1}(u)}{u^{k}}\right|^{m} d u=o(t x)
$$

uniformly in $t$ by (3.1).

We consider now the contribution of the term $S(x, t)$ to the integral on the left of (5.3); that is to say,

$$
\int_{R}^{\infty} d t \int_{0}^{x} x e^{-t x} S(x, t) d x
$$

Given $\varepsilon$, there is an $x_{0}$ such that, for $x>x_{0}$ and all $t>0, S(x, t)<\varepsilon x t$. Hence the contribution of $S(x, t)$ to (5.6) if $x>x_{0}$ is less than

$$
\begin{aligned}
\varepsilon \int_{R}^{\infty} t d t \int_{x_{0}}^{X} x^{2} e^{-t x} d x & <\varepsilon \int_{0}^{X} x^{2} d x \int_{R}^{\infty} t e^{-t x} d t \\
& =\int_{0}^{X}(1+R x) e^{-R x} d x=D \varepsilon X
\end{aligned}
$$


where $D$ is a constant. If $\lambda_{0}>0$ we have $B_{\lambda}^{k-1}(u)=0$ for $u<\lambda_{0}$. If $\lambda_{0}=0$ then the term corresponding to $n=0$ in the sum defining $B_{\lambda}^{k-1}(u)$ vanishes and hence $B_{\lambda}^{k-1}(u)=0$ for $u<\lambda_{1}$. Thus, in any case, there is some $\eta>0$ such that $B_{\lambda}^{k-1}(u)$ $=0$ for $u<\eta$. Thus we have

$$
\left.S(x, t) \leqslant M t \int_{0}^{x}\left|\frac{B_{\lambda}^{k-1}(u)}{u^{k}}\right|^{m} d u=0 \text { for } x<\eta \text { (whatever } t\right) .
$$

Hence the integral $\int_{R}^{\infty} d t \int_{0}^{x_{0}} x e^{-t x} S(x, t) d x$ may be reported by

$$
\int_{R}^{\infty} t d t \int_{\eta}^{x_{0}} x e^{-t x} d x
$$

Once $x_{0}$ has been fixed, the inner integral is less than or equal to $e^{-t \eta} \int_{\eta}^{x_{0}} x d x=$ $c e^{-m}$ ( $c$ is a constant) and this gives the required result.

Let $\mu$ be a number such that $0<m \mu<1$ and also $m \mu<k$. Then if $m>1$, we have $m^{\prime}(1-\mu)>1$, where $\frac{1}{m}+\frac{1}{m^{\prime}}=1$. Now if $v>x$, then, by Hölder's inequality when $m>1$, and trivially when $m=1$, we have

$$
\begin{aligned}
\left|c_{\lambda}^{k}(x)-c_{\lambda}^{k}(v)\right|^{m} & =M\left|\int_{x}^{v} \frac{B_{\lambda}^{k-1}(u)}{u^{k+1}} d u\right|^{m} \\
& \leqslant M x^{m \mu-1} \int_{x}^{v} u^{-m \mu}\left|\frac{B_{\lambda}^{k-1}(u)}{u^{k}}\right|^{m} d u .
\end{aligned}
$$

By (5.7) and Lemma 4,

$$
\begin{aligned}
T(x, t) \leqslant & M t^{k} x^{m \mu-1} \int_{x}^{\infty} u^{-m \mu+k} e^{-t u}\left|\frac{B_{\lambda}^{k-1}(u)}{u^{k}}\right|^{m} d u \\
& +M x^{m \mu-1} \int_{x}^{\infty} u^{-m \mu} e^{-t u}\left|\frac{B_{\lambda}^{k-1}(u)}{u^{k}}\right|^{m} d u \\
& =U(x, t)+V(x, t) .
\end{aligned}
$$

Now

$$
\begin{aligned}
\int_{R}^{\infty} d t \int_{0}^{X} x V(x, t) e^{-t x} d x & \\
\quad= & M \int_{R}^{\infty} d t \int_{0}^{X} x^{m \mu} e^{-t x} d x\left\{\int_{x}^{X}+\int_{X}^{\infty}\right\} u^{-m \mu} e^{-t u}\left|\frac{B_{\lambda}^{k-1}(u)}{u^{k}}\right|^{m} d u \\
& =J_{1}+J_{2} .
\end{aligned}
$$

By changing the order of integration

$$
\begin{aligned}
J_{1} & \leqslant M \int_{R}^{\infty} d t \int_{0}^{X} u^{-m \mu}\left|\frac{B_{\lambda}^{k-1}(u)}{u^{k}}\right|^{m} d u \int_{0}^{\infty} x^{m \mu} e^{-t x} d x \\
& =o(1)\left[\frac{X^{1-m \mu}}{R^{m \mu}}\right]=o(X),
\end{aligned}
$$

by Lemma 5 . 
The inner integral in $J_{2}$, on integration by parts gives in the first place

$$
o\left(X^{1-m \mu} e^{-t X}\right)+o\left\{\int_{X}^{\infty} u\left|d_{u}\left(e^{-t u} u^{-m \mu}\right)\right|\right\} .
$$

Since $e^{-t u} u^{-m \mu}$ is decreasing, we may omit the modulus in the integral if we put a - sign in front; another integration by parts now gives

$$
o\left(X^{1-m \mu} e^{-t X}\right)+o\left\{\int_{X}^{\infty} e^{-t u} e^{-m \mu} d u\right\}
$$

Again by (4.3) the second term is $o\left(X^{-m \mu} e^{-t X} / t\right)$. Since $t X>1$, this may be absorbed in the first term. Hence

$$
\begin{aligned}
& J_{2}=o(X) . \\
& \int_{R}^{\infty} d t \int_{0}^{X} x \cup(x, t) e^{-t x} d x \\
& \quad \leqslant M \int_{R}^{\infty} t^{k} d t \int_{0}^{X} x^{m \mu} e^{-t x} d x\left(\int_{x}^{X}+\int_{X}^{\infty}\right) u^{-m \mu+k} e^{-t u}\left|\frac{B_{\lambda}^{k-1}(u)}{u^{k}}\right|^{m} d u \\
& =I_{1}+I_{2} . \\
& I_{1} \leqslant \int_{R}^{\infty} t^{k} d t \int_{0}^{X} u^{k-m \mu} e^{-t u}\left|\frac{B_{\lambda}^{k-1}(u)}{u^{k}}\right|^{m} d u \int_{0}^{\infty} x^{m \mu} e^{-t x} d x \\
& \quad \leqslant M \int_{0}^{X} u^{k-m \mu}\left|\frac{B_{\lambda}^{k-1}(u)}{u^{k}}\right|^{m} d u \int_{R}^{\infty} t^{k-m \mu-1} e^{-t u} d t
\end{aligned}
$$

But since $0<m \mu<1$, the inner integral is less than

$$
\int_{0}^{\infty} t^{k-m \mu-1} e^{-t u} d t=\frac{\Gamma(k-m \mu)}{u^{k-m \mu}}
$$

and this yields $I_{1}=o(X)$.

Lastly, integrating by parts the inner integral in $I_{2}$, we get

$$
o\left(X^{1+k-m \mu} e^{-t X}\right)+o\left\{\int_{X}^{\infty} u\left|d_{u}\left(u^{k-m \mu} e^{-t u}\right)\right|\right\} .
$$

The expression in curly brackets in (5.14) is equal to

$$
-\int_{X}^{\infty} u d_{u}\left(u^{k-m \mu} e^{-t u}\right)+2 \int_{X}^{(k-m \mu) / t} u d_{u}\left(u^{k-m \mu} e^{-t u}\right),
$$

where the second term is to be omitted in the case $t X>k-m \mu$. The first term may be dealt with in the same way as the corresponding term in the treatment of $J_{2}$, and the second term may be estimated by noting that, uniformly in the range of integration, $u=O\left(\frac{1}{t}\right)$. We find that the expression in (5.14) is $o\left(X^{1+k-m \mu} e^{-t X}\right)$. 
Thus we now get

$$
\begin{aligned}
I_{2} & =o\left\{X^{1+k-m \mu} \int_{R}^{\infty} t^{k} e^{-t X} d t \int_{0}^{X} x^{m \mu} e^{-t x} d x\right\} \\
& =o\left\{X^{1+k-m \mu} \int_{0}^{\infty} t^{k} e^{-t X} d t \int_{0}^{\infty} x^{m \mu} e^{-t x} d x\right\} \\
& =o\left\{X^{1+k-m \mu} \int_{0}^{\infty} t^{k-m \mu-1} e^{-t X} d t\right\} \\
& =o(X),
\end{aligned}
$$

since $k>m \mu$. Combining all these (5.4) to (5.15) we get (5.3).

This completes the proof of the theorem.

Thanks are due to Professor B. Kuttner for his valuable suggestions on the revision of the paper and to the referee for his valuable comments.

\section{REFERENCES}

1. A. V. Boyd and J. M. Hyslop, A definition of strong Rieszian summability and its relationship to strong Cesàro summability, Proc. Glasgow Math. Assoc. 1 (1952), 94-99.

2. K. Chandrasekharan and S. Minakshisundaran, Typical means, Tata Inst. of Fundamental Research Monograph on Math., no. 1, Oxford Univ. Press, 1952.

3. T. M. Flett, Some generalizations of Tauber's second theorem, Quart. J. Math. Oxford Ser. (2) 10 (1959), 70-80.

4.

5. M. Glatfeld, On strong Rieszian summability, Proc. Glasgow Math. Assoc. 3 (1957), 123-131.

6. G. H. Hardy and M. Riesz, The general theory of Dirichlet's series, Cambridge Tracts in Math. and Math. Phys., no. 18, Stechert-Hafner, New York, 1964.

7. P. Srivastava, On strong Rieszian summability of infinite series, Proc. Nat. Inst. Sci. India Part A 23 (1957), 58-71.

8. , On strong summability of infinite series, Math. Student 31 (1963), 167-192.

Department of Mathematics, Sambalpur University, Jyoti Vihar, Burla, 768017, India 